\title{
Oocyte quality improvement using a herbal medicine comprising 7 crude drugs
}

\author{
Taketo Inoue ${ }^{1,2^{*}}$, Yoshiyuki Ono ${ }^{1}$, Yukiko Yonezawa ${ }^{1}$, Michinobu $\mathrm{Oi}^{3}$, Naomi Kobayashi ${ }^{1}$, Junji Kishi ${ }^{1}$, \\ Nobuyuki Emi ${ }^{1}$ \\ ${ }^{1}$ Ono Ladies Clinic, Hyogo, Japan \\ ${ }^{2}$ Department of Biophysics, Graduate School of Health Science, Kobe University, Kobe, Japan \\ ${ }^{3}$ Herb Pharmacy Co., Ltd., Kobe, Japan

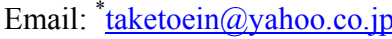

Received 11 December 2012; revised 13 January 2013; accepted 21 January 2013

\begin{abstract}
Maternal age is a significant factor in infertility treatment. Ovarian function and oocyte quality decrease with age, whereas the frequency of chromosomal abnormalities increases. In this study, improvement of oocyte quality and ovarian function were attempted using a herbal medicine comprising 7 crude drugs: Angelicae radix, Rehmanniae radix, Plantaginis semen, Lonicerae flos, Carthami flos, Ginseng radix, and $\mathrm{Cu}$ curbita moschata Duch. Thirty-one women who repeatedly failed to conceive by intracytoplasmic sperm injection took the herbal medicine before breakfast and dinner from the start of menstrual cycle in the ovum pickup cycle. Average patient age was $38.5 \pm$ 0.7 years, and the average ovum pickup frequency on the first dosage day was $7.9 \pm 1.5$. To analyze the effects of herbal medicine intake, the number of recovered and mature oocytes, their morphology and physical qualities, as well as the rates of fertilization, oocyte development, and pregnancy was compared before and after intake. The recovered and mature oocyte numbers, oocyte morphology and physical qualities, and fertilization rate were not significantly different before and after drug intake. However, the oocyte development rate was significantly higher $(58.0 \%)$ after herbal medicine intake than before $(32.5 \%$; $p=$ 0.0003). Moreover, the successful pregnancy rate was significantly higher after intake than before $(6.9 \%$ versus $0 \% ; p=0.0111)$. Herbal medicine may constitute a useful adjunct to assisted reproductive technology in women.
\end{abstract}

Keywords: Herbal Medicine; Improvement of the Oocyte Development Rate; Intracytoplasmic Sperm Injection; Oocyte Quality; Repeated Infertility Treatment Failure

"Corresponding author.

\section{INTRODUCTION}

The current average maternal age at first childbirth has increased compared with that in the 1970s. Primiparous women are now often in their late twenties, reflecting a shift in the timing of family formation [1]. Consequently, use of infertility treatment has increased.

Maternal age is a significant factor in infertility treatment. Ovarian function and oocyte quality decrease with age, whereas the frequency of chromosomal abnormalities increases. Accordingly, fertilization, development, and pregnancy rates decrease, whereas the spontaneous abortion rate increases [2-4]. Oocyte quality markedly decreases in women aged $>35$ years and chromosomal aneuploidy increases: correspondingly, the implantation rate following embryo transfer diminishes $[5,6]$.

Oocyte quality is an important prognostic factor, because nuclear and cytoplasmic oocyte maturity may directly relate to the success of intracytoplasmic sperm injection (ICSI). Dysmorphic oocytes, i.e., those exhibiting centrally located granular cytoplasm (CLGC) [7], vacuolization [8], or smooth endoplasmic reticulum clusters (sERC) $[9,10]$, are frequently observed [11]. During piezo-ICSI, low-stretching oocytes, which penetrate before piezo pulse application, are also common [12]. Previous studies [7-12] report low pregnancy rates using fertilized eggs derived from oocytes of abnormal morphology or low quality.

Several studies attempted to improve oocyte quality and ovum function by adjuncts to assisted reproductive technology (ART), such as dehydroepiandrosterone [13, 14] or herbal medicine [15-17] administration, low reactive-level laser therapy (LLLT) [18], or acupuncture [19]. Reportedly [15,17,20,21], successful pregnancies were achieved after herbal medicine intake. Recently, it was reported $[17,20,21]$ that women who repeatedly failed to conceive by ART became pregnant after macrophageactivating Chinese mixed herbs (MACH) intake. MACH comprises Plantaginis semen, Cucurbita moschata Duch, 
Lonicerae flos, and Carthami flos; it is known to ameliorate the clinical phenomena associated with menopause [22]. Therefore, improvement of oocyte quality and ovarian function were attempted by MACH administration at our clinic. In a previous study (Inoue et al., unpublished data), 18 women (average age: 39.8 years; range: 35 - 46 years) who repeatedly failed to conceive by ART took $\mathrm{MACH}$. However, the oocyte development and successful pregnancy rate did not improve in this study (rate of developed blastocysts before versus after dosage: $23.8 \%$ versus $34.4 \%, p=0.26$; successful pregnancy rate before versus after dosage: $0 \%$ versus $0 \%$ ).

With adjuncts to ART such as LLLT and acupuncture, ovarian function and blood flow reportedly improve, consequently increasing oocyte number and pregnancy rate [13-16,18,19]. Accordingly, the composition of MACH was changed, adding Angelicae radix and Rehmanniae radix, which improve blood flow, and Ginseng radix, which has nutritional benefits and improves blood flow. Angelicae radix is frequently used in the field of obstetrics and gynecology and is often taken with Rehmanniae radix. Zhang et al. [23] reported that oral administration of Z-ligustilide (LIG), extracted from Angelicae radix, decreased thrombus formation in rats similarly to aspirin. Zhu et al. [24] suggested that catalpol, extracted from Rehmanniae radix, regulates angiogenesis in the brains of rats after stroke by increasing erythropoietin and vascular endothelial growth factor (VEGF), thereby decreasing ischemic neuronal damage and enhancing functional recovery. Ginseng radix reportedly $[25,26]$ improves poor peripheral circulation and is also used for its restorative and health-promoting properties [25,27]. Moreover, saponins extracted from Ginseng radix have been shown to have analeptic properties and they enhance responses to viral infection, tumor load, temperature stress, and exercise-induced trauma [25,27].

In this study, improvement of oocyte quality and ovarian function was attempted by herbal medicine intake.

\section{MATERIALS AND METHODS}

\subsection{Herbal Medicine}

A herbal medicine comprising 7 crude drugs $-0.7 \mathrm{~g} \mathrm{An-}$ gelicae radix, $0.3 \mathrm{~g}$ Rehmanniae radix, $0.7 \mathrm{~g}$ Plantaginis semen, $0.7 \mathrm{~g}$ Lonicerae flos, $0.7 \mathrm{~g}$ Carthami flos, $0.3 \mathrm{~g}$ Ginseng radix, and $0.7 \mathrm{~g}$ Cucurbita moschata Duchwas prepared as pills with $1.5 \mathrm{~g} \mathrm{Mel}$. Informed consent was obtained regarding the dosage of herbal medicine. All procedures were performed according to the Declaration of Helsinki.

\subsection{Patients}

A retrospective before-after study was performed on data obtained from ovum pickup cycles after herbal medicine administration between January 2009 and May 2012 (174 cycles). Thirty-one women consumed the herbal medicine before breakfast and dinner from the start of menstruation in the ovum pickup cycle. Average patient age was $38.5 \pm 0.7$ years and the average ovum pickup frequency on the first day was $7.9 \pm 1.5$. Male patients were categorized on the basis of motile spermatozoa number as follows: normal $\left(>20 \times 10^{6}\right.$ spermatozoa $/ \mathrm{ml} ; n$ $=12)$; mild oligospermia $\left(>10-20 \times 10^{6}\right.$ spermato$\mathrm{zoa} / \mathrm{ml} ; n=8)$; and severe oligospermia $\left(<5 \times 10^{6}\right.$ spermatozoa $/ \mathrm{ml} ; n=11$ ) (Table 1). Normal patients and those with mild oligospermia underwent 2 in vitro fertilization (IVF) cycles before ICSI; however, the success rate of IVF was low $(0 \%-16.6 \%)$.

\subsection{Ovarian Stimulation/Intracytoplasmic Sperm Injection}

For ovarian stimulation, either the short or gonadotropinreleasing hormone $(\mathrm{GnRH})$ antagonist protocols were used, depending on the patient. In poor responders, clomifene citrate $(50 \mathrm{mg}$ Spacromin tablet; Pola Pharma Inc., Tokyo, Japan) and human menopausal gonadotropin (hMG; ASKA Pharmaceutical Co., Ltd., Tokyo, Japan) were used for ovarian stimulation. GnRH analogue acetate (Fuji Pharma Co., Ltd., Tokyo, Japan), human menopausal gonadotrophin, and GnRH antagonist (Cetrotide ${ }^{\mathbb{B}}$; Shionogi \& Co., Ltd., Osaka, Japan) were used in both protocols. When at least 2 follicles reached $18-20 \mathrm{~mm}$ in diameter (determined by transvaginal ultrasound), 5000 IU of human chorionic gonadotropin (hCG; Fuji Pharma) was administered. Ovum pickup was performed $35 \mathrm{~h}$ after hCG injection. Oocytes were freed from cumulus cells using $0.025 \%$ hyaluronidase (Sigma-Aldrich Corp., St. Louis, MO, USA) dissolved in Universal IVF Medium (MediCult, Jyllinge, Denmark). After denudation, metaphase II (MII) oocytes were cultured for $\geq 2 \mathrm{~h}$. Oocytes were inseminated by ICSI in Universal IVF me-

Table 1. Patients' characteristics on first dosage day.

\begin{tabular}{lc}
\hline & Characteristics of participants \\
\hline Number of couples & 31 \\
Female & $38.5 \pm 0.7$ \\
Age & $7.9 \pm 1.5$ \\
OPU frequency & \\
Male & 12 \\
Number of normozoospermia & 8 \\
Number of mildoligospermia & 11 \\
Number of severe oligospermia & \\
\hline
\end{tabular}

Normal: $>20 \times 10^{6}$ spermatozoa/ml; mild oligospermia: $>10-20 \times 10^{6}$ spermatozoa $/ \mathrm{ml}$; severe oligospermia: $<5 \times 10^{6}$ spermatozoa $/ \mathrm{ml}$. 
dium $3 \mathrm{~h}$ after pickup. Before ICSI, oocytes underwent investigation for morphology and quality on the basis of the presence or absence of CLGC, vacuolization, and sERC.

Piezo-ICSI was performed with both injection (Prime Tech Ltd., Ibaraki, Japan) and holding (Kitazato BioPharma Co. Ltd., Shizuoka, Japan) pipettes using a micromanipulator (Narishige Scientific Instrument Laboratory, Tokyo, Japan) attached to a piezo impact drive unit (PMM-150/FU; Prime Tech Ltd.) under Hoffman modulation optics, according to Kimura and Yanagimachi [28]. Ova in Universal IVF Medium under mineral oil (Irvine Scientific, Santa Ana, CA, USA) underwent ICSI after sperm selection by hyaluronic acid (HA) [29]. Several HA-bound spermatozoa were picked up, washed in $7 \%$ polyvinylpyrrolidone solution with human serum albumin (Irvine Scientific), and immobilized. One spermatozoon was aspirated, tail first, into an injection pipette after immobilization by the application of several piezo pulses (speed: 3; intensity: 3 ). The oocyte to be injected was secured with a holding pipette (nine o'clock position) adjacent to the polar body (6 o'clockor 12 o'clock position). The zona pellucida was penetrated by several piezo pulses (speed: 3; intensity: 3 ) at the 3 o'clock position. After the spermatozoon was repositioned at the tip of the injection pipette, it was inserted deep into the oocyte by stretching the oolemma, and a single piezo pulse (speed: 1 ; intensity: 1 or 2) was applied to penetrate the oolemma. The spermatozoon was injected into the ooplasm. The pipette was withdrawn gently and the oocyte was released from the holding pipette. The following morning, fertilization was confirmed by the presence and number of pronuclei and polar bodies, and the fertilized eggs were cultured in Single Step Medium ${ }^{\mathrm{TM}}$ (Irvine Scientific), including 10\% serum substitute supplement (Irvine Scientific), until Days 2 - 5.

Oocyte stretchability was confirmed by use of a mechanically driven, blunt pipette, according to Iwayama et al. [12]. Oolemma were penetrated either before the application of a piezo pulse at $\leq 75 \%$ stretch (low stretchability) or by a piezo pulse when the oolemma was stretched $>75 \%$ (high stretchability). Individual oocyte diameter was considered $100 \%$ for the determination of penetration position.

\subsection{Fresh Embryo Transfer}

A cleaved embryo or morula was transferred to the uterus on Days 2, 3, or 4. For luteal support during the fresh embryo transfer cycle, patients were administered $81 \mathrm{mg}$ acetylsalicylic acid (Bufferin combination tablet A81; Lion Corporation, Tokyo, Japan) and a $200 \mathrm{mg}$ progesterone vaginal suppository once daily from the day after ovum pickup until the urine hCG test. Progesterone va- ginal suppositories were made by dissolving progesterone (Sigma-Aldrich Corp.) and Macrogol 6000 (Nikko Chemicals Co., Ltd., Tokyo, Japan) in Macrogol 400 (Yoshida Pharmaceutical Co., Ltd., Tokyo, Japan) [30].

On the day of embryo transfer, if serum progesterone concentration was $<15 \mathrm{ng} / \mathrm{ml}$, women were injected with $125 \mathrm{mg} 17 \alpha$-hydroxyprogesterone caproate (depot progesterone intramuscular injection; Fuji Pharma) once every 5 days.

\subsection{Cryopreservation/Thawing/Frozen-Thawed Embryo Transfer}

Blastocysts were cryopreserved and thawed using the Cryotop technique, as described by Kuwayama [31], except that thawing was performed at room temperature.

A frozen-thawed blastocyst was transferred to the uterus. For luteal support during frozen-thawed embryo transfer cycles, patients were administered $2 \mathrm{mg}$ chlormadinone acetate (Lutoral; Shionogi \& Co. Ltd.) 3 times daily from ovulation, and $0.72 \mathrm{mg}$ transdermal estradiol patches (Estran $a^{\circledR}$ Tape; Hisamitsu Pharmaceutical Co. Inc., Tokyo, Japan) were applied to the abdomen from the start of menstruation for 10 weeks. On the day of embryo transfer, if serum progesterone concentration was $<15 \mathrm{ng} / \mathrm{ml}$, women were injected with $125 \mathrm{mg} 17 \alpha$-hydroxyprogesterone caproate once every 5 days.

\subsection{Statistical Analysis}

Statistical analysis was performed using the chi-square and Fisher's exact tests. $\mathrm{p}<0.05$ and $\mathrm{p}<0.01$ were considered statistically significant.

\section{RESULTS}

The present study investigated improvement in oocyte quality and ovarian function after herbal medicine intake. Recovered and mature oocyte numbers were similar before and after herbal medicine intake (Table 2). In patients with premature menopause, ovum pickup after herbal medicine intake was attempted twice, but no oocytes were obtained (data not shown). No significant differences in oocyte morphology and physical qualities (Table 3) or fertilization rate (Table 4) were evident before and after herbal medicine intake. Moreover, no significant difference in the oocyte degeneration rate before and after herbal medicine intake was observed (Table 4).

To investigate fertilized oocyte development, embryo culture, fresh embryo transfer, and frozen-thawed blastocyst transfer were performed. The number of fertilized eggs that developed into blastocysts was significantly higher after herbal medicine intake than before [ 47 blastocysts/81 fertilized eggs (58.0\%) versus 40/123 (32.5\%); $\mathrm{p}=0.0003$; Table 4]. 
Table 2. Oocyte maturation.

\begin{tabular}{|c|c|c|c|c|c|}
\hline & \multicolumn{4}{|c|}{ Intake of herbal medicine } & \multirow{2}{*}{$\mathrm{p}$ value } \\
\hline & \multicolumn{2}{|c|}{ Before } & \multicolumn{2}{|c|}{ After } & \\
\hline \multicolumn{6}{|l|}{ Ovumpickup } \\
\hline No. of recovered oocytes & 376 & & 224 & & \\
\hline No. of MII oocytes & 298 & $(79.3 \%)$ & 180 & $(80.4 \%)$ & 0.9086 \\
\hline No. of MI oocytes & 15 & $(4.0 \%)$ & 7 & $(3.1 \%)$ & 0.9965 \\
\hline No. of GV oocytes & 18 & $(4.8 \%)$ & 13 & $(5.8 \%)$ & 0.6039 \\
\hline No. of degenerated oocytes & 45 & $(12.0 \%)$ & 24 & $(10.7 \%)$ & 0.6149 \\
\hline Average recovered oocytes & \multicolumn{2}{|c|}{$3.5 \pm 3.0$} & \multicolumn{2}{|c|}{$3.3 \pm 2.3$} & \\
\hline
\end{tabular}

Abbreviations: MII, metaphase II; MI, metaphase I; GV, germinal vesicle. Based on the chi-square test, $\mathrm{p}<0.05$ was considered statistically significant.

Table 3. Oocyte morphology and physical qualities.

\begin{tabular}{|c|c|c|c|c|c|}
\hline & \multicolumn{4}{|c|}{ Intake of herbal medicine } & \multirow{2}{*}{$\mathrm{p}$ value } \\
\hline & \multicolumn{2}{|c|}{ before } & \multicolumn{2}{|c|}{ after } & \\
\hline No. of MII oocytes & 298 & & 180 & & \\
\hline No. of sERCs $(+)$ oocytes & 2 & $(0.7 \%)^{*}$ & 6 & $(3.3 \%)^{*}$ & 0.0283 \\
\hline No. of vacuoles $(+)$ oocytes & 4 & $(1.3 \%)$ & 5 & $(2.8 \%)$ & 0.2655 \\
\hline No. of CLGC $(+)$ oocytes & 44 & $(14.8 \%)$ & 33 & $(18.3 \%)$ & 0.3113 \\
\hline \multicolumn{6}{|l|}{ Physical quality } \\
\hline No. of MII oocytes & 298 & & 180 & & \\
\hline High stretchability & 238 & $(79.9 \%)$ & 138 & $(76.7 \%)$ & 0.4081 \\
\hline Low stretchability & 60 & $(20.1 \%)$ & 42 & $(23.3 \%)$ & 0.4081 \\
\hline
\end{tabular}

Abbreviations: MII, metaphase II; sERC, smooth endoplasmic reticulum clusters; CLGC, centrally located granular cytoplasm. *: significantly different between groups (using the chi-square test, where $\mathrm{p}<0.05$ was considered statistically significant).

Table 4. Fertilization and oocyte development rates.

\begin{tabular}{|c|c|c|c|c|c|}
\hline & \multicolumn{4}{|c|}{ Intake of herbal medicine } & \multirow{2}{*}{$\mathrm{p}$ value } \\
\hline & \multicolumn{2}{|c|}{ Before } & \multicolumn{2}{|c|}{ After } & \\
\hline No. of MII oocytes & 298 & & 180 & & \\
\hline No. of 2PN2PB oocytes & 188 & $(63.1 \%)$ & 129 & $(71.7 \%)$ & 0.0545 \\
\hline No. of 1PN2PB oocytes & 12 & $(4.0 \%)$ & 10 & $(5.6 \%)$ & 0.4444 \\
\hline No. of 3PN1PB oocytes & 0 & $(0.0 \%)$ & 2 & $(1.1 \%)$ & 0.0687 \\
\hline No. of OPN2PB oocytes & 35 & $(11.7 \%)^{*}$ & 10 & $(5.6 \%)^{*}$ & 0.0197 \\
\hline No. of unfertilized oocytes & 44 & $(14.8 \%)$ & 22 & $(12.2 \%)$ & 0.3708 \\
\hline No. of degenerate oocyte after ICSI & 19 & $(6.4 \%)$ & 7 & $(3.9 \%)$ & 0.2134 \\
\hline \multicolumn{6}{|l|}{ Culture } \\
\hline No. of cultured oocytes & 123 & & 81 & & \\
\hline No. of blastocysts & 40 & $(32.5 \%)^{* *}$ & 47 & $(58.0 \%)^{* *}$ & 0.0003 \\
\hline
\end{tabular}

Abbreviations: MII, metaphase II; PN, pronucleus; PB, polar body. *: significantly different between groups (using the chi-square test, where $\mathrm{p}<0.05$ was considered statistically significant). ${ }^{* *}$ : significantly different between groups (using the chi-square test, where $\mathrm{p}<0.01$ was considered statistically significant). 
In pregnancies following embryo transfer without herbal medicine intake, gestational sacs were evident on transvaginal ultrasound, but fetal heartbeats were absent. However, in pregnancies after herbal medicine intake, both gestational sacs and fetal heartbeats were observed (Table 5). The successful pregnancy rate was significantly higher after herbal medicine intake than before [5 successful pregnancies/72 embryo transfers (6.9\%) versus $0 / 90(0 \%) ; p=0.0111$; Table 5]. No structural abnormalities were evident during routine checkups. Three babies were born: they were healthy when examined during the second and fourth weeks after birth (Table 5). Regarding fresh embryo transfer, the successful pregnancy rate was not improved by herbal medicine intake (Table 5); however, regarding frozen-thawed embryo transfer, the successful pregnancy rate was higher after herbal medicine intake than before [4 successful pregnancies/30 embryo transfers $(13.3 \%)$ versus $0 / 30(0 \%)$; $=0.0562$; Table 5].

No adverse effects of the herbal medicine were observed. In the 3 successful pregnancies, all mothers and babies were healthy. Furthermore, several patients undergoing infertility treatments, such as artificial insemination and IVF, also consumed the herbal medicine. No side effects were observed in these patients and their babies (data not shown).

\section{DISCUSSION}

The present study showed that development of fertilized eggs into blastocysts improved following intake of a herbal medicine comprising 7 crude drugs: Angelicae radix, Rehmanniae radix, Plantaginis semen, Lonicerae flos, Carthami flos, Ginseng radix, and Cucurbita moschata Duch. Consequently, the successful pregnancy rate significantly increased.

Recovered oocyte number was similar before $(3.5 \pm 3$ oocytes) and after $(3.3 \pm 2.3$ oocytes $)$ herbal medicine intake. However, when the data were separated by patient, ovarian function improvement was evident. In 1 patient, the average recovered oocytes was 1 before intake and 3.5 after; in another, the average recovered oocytes was 4.5 before intake and 8 after. Thus, the effect of herbal medicine varies between individuals.

Nuclear maturity may directly relate to the success rate of ICSI. Rienzi et al. [32] reported that $7.9 \%$ of cumulus-oocyte complexes contained germinal vesiclestage (GV) oocytes; 5.6\% contained metaphase I (MI)

Table 5. Embryo transfer outcomes.

\begin{tabular}{|c|c|c|c|c|c|}
\hline \multirow{2}{*}{ Embryo transfer (Fresh and Frozen) } & \multicolumn{4}{|c|}{ Intake of herbal medicine } & \multirow{2}{*}{$\mathrm{p}$ value } \\
\hline & \multicolumn{2}{|c|}{ before } & \multicolumn{2}{|c|}{ After } & \\
\hline No. of fresh embryo transfers & 90 & & 72 & & \\
\hline No. of chemical pregnancies & 8 & $(8.9 \%)$ & 9 & $(12.5 \%)$ & 0.4561 \\
\hline No. of clinical pregnancies & 4 & $(4.4 \%)$ & 6 & $(8.3 \%)$ & 0.3067 \\
\hline No. of fetuses & 0 & $(0.0 \%)^{*}$ & 5 & $(6.9 \%)^{*}$ & 0.0111 \\
\hline No. of live births and pregnancies & 0 & $(0.0 \%)^{*}$ & $5(2)^{\mathrm{a}}$ & $(6.9 \%)^{*}$ & 0.0111 \\
\hline \multicolumn{6}{|l|}{ Fresh embryo transfer } \\
\hline No. of fresh embryo transfers & 60 & & 42 & & \\
\hline No. of chemical pregnancies & 6 & $(10.0 \%)$ & 1 & $(2.4 \%)$ & 0.1348 \\
\hline No. of clinical pregnancies & 3 & $(5.0 \%)$ & 1 & $(2.4 \%)$ & 0.4530 \\
\hline No. of fetuses & 0 & $(0.0 \%)$ & 1 & $(2.4 \%)$ & 0.4174 \\
\hline No. of live births & 0 & $(0.0 \%)$ & 1 & $(2.4 \%)$ & 0.4174 \\
\hline \multicolumn{6}{|l|}{ Frozen-thawed embryo transfer } \\
\hline No. of frozen-thawed embryo transfer & 30 & & 30 & & \\
\hline No. of chemical pregnancies & 2 & $(6.7 \%)^{*}$ & 8 & $(26.7 \%)^{*}$ & 0.0399 \\
\hline No. of clinical pregnancies & 1 & $(3.3 \%)$ & 5 & $(16.7 \%)$ & 0.0973 \\
\hline No. of fetuses & 0 & $(0.0 \%)$ & 4 & $(13.3 \%)$ & 0.0562 \\
\hline No. of live births and pregnancies & 0 & $(0.0 \%)$ & $4(2)^{\mathrm{a}}$ & $(13.3 \%)$ & 0.0562 \\
\hline
\end{tabular}


oocytes, and $79.8 \%$ contained MII oocytes. Proportions of GV, MI, and MII oocytes before and after herbal medicine intake corresponded with this study. Moreover, our results showed that nuclear maturity was similar before and after herbal medicine intake. Therefore, the effect of herbal medicine on oocyte nuclear maturity is unconfirmed by this study.

In previous studies, women with low-quality oocytes judged unsuitable for ICSI began producing good quality oocytes and, subsequently, became pregnant after taking MACH [20,21]. Our herbal medicine was based on $\mathrm{MACH}$, which comprises Plantaginis semen, Carthami flos, Lonicerae flos, and Cucurbita moschata Duch. Each of the 4 crude drugs induces inferferon [17]. MACH modulates the macrophage function and is involved in the production of immunoglobulin E antibody [17]. After $\mathrm{MACH}$ intake, blood pressure and skin surface blood flow decreased [22]. Moreover, plasma follicle-stimulating hormone (FSH) concentration decreased in case with high basal plasma FSH under MACH administration [17]. Therefore, MACH is involved in maintenance of homeostasis such as the immune system and endocrinological regulation systems. As a result of modulated homeostasis, $\mathrm{MACH}$ ameliorates clinical phenomena associated with menophase and improves oocytes quality. To these crude drugs, Angelicae radix, Rehmanniae radix, and Ginseng radix were added. This herbal medicine was administered to women with low-quality oocytes judged unsuitable for ICSI in an attempt to improve oocyte quality. However, good quality oocytes were not obtained and the percentage of degenerated oocytes remained unchanged.

Iwayama et al. [12] demonstrated that stretchability of the human oolemma during piezo-ICSI affects postinjection survival, but not the potential to develop into blastocysts. In the present study, the proportion of oocytes with low stretchability corresponded with this report (19.1\%) [12]. Oolemma stretchability was not increased by herbal medicine intake and the rate of degenerated oocytes following ICSI was unchanged. Therefore, it is implausible that oolemma stretchability underlies the improved blastocyst number evident in this study. Morphological qualities, except sERC, were not significantly different before and after herbal medicine intake. Otsuki et al. [10] reported that serum estradiol (E2) levels on the day of hCG administration were significantly higher in sERC-positive cycles, suggesting that high E2 levels cause sERC formation. In this study, 23 patients were poor responders to ovarian stimulation, despite the rate of sERC formation significantly increased after herbal medicine intake: 5 sERC-positive oocytes were removed from 1 patient. These results suggest that E2 levels on the day of hCG administration may increase upon herbal medicine intake.
Previous reports [7] demonstrated a CLGC frequency of $17.3 \%-65.8 \%$, although discrepancies exist in the data obtained from these studies. Regarding vacuole frequency, our data showed an approximate value similar to that reported by Meriano et al. [9]. Therefore, herbal medicine intake did not demonstrate an effect on oocyte morphology and physical qualities.

Fertilization rates were not improved by herbal medicine administration, but the number of fertilized eggs that developed into blastocysts increased. Ushiroyama et al. [21] also reported an improvement in the number of fertilized eggs that developed into blastocysts using MACH. In our preliminary experiment, improvement of the pregnancy rate was attempted using $\mathrm{MACH}$; however, the oocyte development and pregnancy rates showed no change in 18 women (Inoue et al., unpublished data). Accordingly, this preliminary experiment was suspended at our clinic. Several studies attempted to improve blood flow in the body, including that in the ovary, using herbal medicine [15,16], LLLT [18,33] or acupuncture [19]. Iwahata et al. [33] reported a significantly higher pregnancy rate with ICSI-LLLT than ICSI alone. The authors believe that LLLT improves oocyte quality by increasing blood flow. Acupuncture may improve ovulation by the modulation of the central and peripheral nervous systems, neuroendocrine and endocrine systems, ovarian blood flow, and metabolism. When embryo transfer was performed during acupuncture, pregnancy rate improved [19]. Angelicae radix, Rehmanniae radix, and Ginseng radix were added to MACH. LIG, extracted from Angelicae radix, causes a dose-dependent decrease in thrombus formation and adenosine diphosphate-induced platelet aggregation [23]. Catalpol, extracted from Rehmanniae radix, up regulate serythropoietin and VEGF, thereby modulating angiogenesis [24]. Furthermore, Ginseng radix improves poor peripheral circulation [26]. Therefore, blood flow could underlie oocyte quality and ovum function: improving blood flow increased the number of eggs that developed into blastocysts and the successful pregnancy rate. Subsequently, improved blood flow may contribute to the continuation of pregnancy.

The present study shows that herbal medicine can be a useful adjunct to ART in women who repeatedly fail to conceive by infertility treatment.

\section{REFERENCES}

[1] Organisation for Economic Cooperation and Development (2011) OECD family database. www.oecd.org/social/family/database.

[2] Baird, D.T., Collins, J., Egozcue, J., Evers, L.H., Gianaroli, L., Leridon, H., Sunde, A., Templeton, A., Van Steirteghem, A., Cohen, J., Crosignani, P.G., Devroey, P., Diedrich, K., Fauser, B.C., Fraser, L., Glasier, A., Liebaers, 
I., Mautone, G., Penney, G., Tarlatzis, B. and ESHRE Capri Workshop Group (2005) Fertility and aging. Human Reproduction Update, 11, 261-276. doi:10.1093/humupd/dmi006

[3] Practice Committee of the American Society for Reproductive Medicine (2002) Aging and infertility in women: A committee opinion. Fertility and Sterility, 78, 215-219. doi:10.1016/S0015-0282(02)03212-0

[4] Djahanbakhch, O., Ezzati, M. and Zosmer, A. (2007) Reproductive aging in women. Journal of Pathology, 211, 219-231. doi:10.1002/path.2108

[5] Pellestor, F., Andréo, B., Arnal, F., Humeau, C. and Demaille, J. (2003) Maternal aging and chromosomal abnormalities: New data drawn from in vitro unfertilized human oocytes. Human Genetics, 112, 195-203.

[6] te Velde, E.R. and Pearson, P.L. (2002) The variability of female reproductive aging. Human Reproduction Update, 8, 141-154. doi:10.1093/humupd/8.2.141

[7] Kahraman, S., Yakın, K., Dönmez, E., Şamlı, H., Bahçe, M., Cengiz, G., Sertyel, S., Şamlı, M. and İmirzalıoğlu, N. (2000) Relationship between granular cytoplasm of oocytes and pregnancy outcome following intracytoplasmic sperm injection. Human Reproduction, 15, 2390-2393. doi:10.1093/humrep/15.11.2390

[8] Ebner, T., Moser, M., Sommergruber, M., Gaiswinkler, U., Shebl, O., Jesacher, K. and Tews, G. (2005) Occurrence and developmental consequences of vacuoles throughout preimplantation development. Fertility and Sterility, 83, 1635-1640. doi:10.1016/j.fertnstert.2005.02.009

[9] Meriano, J.S., Alexis, J., Visram-Zaver, S., Cruz, M. and Casper, R.F. (2001) Tracking of oocyte dysmorphisms for ICSI patients may prove relevant to the outcome in subsequent patient cycles. Human Reproduction, 16, 21182123. doi:10.1093/humrep/16.10.2118

[10] Otsuki, J., Okada, A., Morimoto, K., Nagai, Y. and Kubo, H. (2004) The relationship between pregnancy outcome and smooth endoplasmic reticulum clusters in MII human oocytes. Human Reproduction, 19, 1591-1597. doi:10.1093/humrep/deh258

[11] Van Blerkom, J. and Henry, G. (1992) Oocyte dysmorphism and aneuploidy in meiotically mature human oocytes after ovarian stimulation. Human Reproduction, 7, 379-390.

[12] Iwayama, H., Hochi, S. and Yamashita, M. (2010) Low stretching ability of human oolemma during piezo-ICSI as a risk factor on post-injection survival and implantation. Journal of Mammalian Ova Research, 27, 150-156. doi:10.1274/jmor.27.150

[13] Barad, D.H. and Gleicher, N. (2005) Increased oocyte production after treatment with dehydroepiandrosterone. Fertility and Sterility, 84, 756. doi:10.1016/j.fertnstert.2005.02.049

[14] Wiser, A., Gonen, O., Ghetler, Y., Shavit, T., Berkovitz, A. and Shulman, A. (2010) Addition of dehydroepiandrosterone (DHEA) for poor-responder patients before and during IVF treatment improves the pregnancy rate: A randomized prospective study. Human Reproduction, 25, 2496-2500. doi:10.1093/humrep/deq220
[15] Chao, S.L., Huang, L.W. and Yen, H.R. (2003) Pregnancy in premature ovarian failure after therapy using Chinese herbal medicine. Chang Gung Medical Journal, 26, 449-452.

[16] Zhou, J. and Qu, F. (2009) Treating gynecological disorders with traditional Chinese medicine: A review. African Journal of Traditional, Complementary, and Alternative Medicines: AJTCAM/African Networks on Ethnomedicines, 6, 494-517.

[17] Ushiroyama, T., Yokoyama, N., Hakukawa, M., Sakuma, K., Ichikawa, F. and Yoshida, S. (2012) Clinical efficacy of macrophage-activating Chinese mixed herbs (MACH) in improvement of embryo qualities in women with longterm infertility of unknown etiology. The American Journal of Chinese Medicine, 40, 1-10. doi:10.1142/S0192415X12500012

[18] Fujii, S., Ohshiro, T., Ohshiro, T., Sasaki, K. and Taniguchi, Y. (2007) Proximal priority treatment using the neck irradiator for adjunctive treatment of female infertility. Laser Therapy, 16, 133-136. doi: $10.5978 /$ islsm.16.133

[19] Huang, D.M., Huang, G.Y., Lu, F.E., Stefan, D., Andreas, N. and Robert, G. (2011) Acupuncture for infertility: Is it an effective therapy? Chinese Journal of Integrative Medicine, 17, 386-395. doi:10.1007/s11655-011-0611-8

[20] Kumagai, A., Yokoyama, N., Ichikawa, F. and Ushiroyama, T. (2009) Attempt of embryo quality improvement by macrophage activation Chinese mixed herbs $(\mathrm{MACH})$ to patients who repeatedly fail in in-vitro fertilization. Journal of Clinical Embryologist, 11, 7-11.

[21] Ushiroyama, T., Kumagai, A., Yokoyama, N., Hakukawa, M., Sakuma, K. and Ichikawa, F. (2009) Clinical application of macrophage activating Chinese mixed herbs to long-term sterile women with unknown cause. Obstetrical and Gynecological Therapy, 98, 191-196.

[22] Ushiroyama, T., Yoshida, S., Tadaki, K., Ikeda, A. and Ueki, M. (2004) Clinical efficacy of EH0202, a Kampo formula, on the health of middle-aged women. The American Journal of Chinese Medicine, 32, 755-770. doi:10.1142/S0192415X04002314

[23] Zhang, L, Du, J.R., Wang, J., Yu, D.K., Chen, Y.S., He, Y. and Wang, C.Y. (2009) Z-ligustilide extracted from Radix Angelica Sinensis decreased platelet aggregation induced by ADP ex vivo and arterio-venous shunt thrombosis in vivo in rats. Yakugaku Zasshi, 129, 855-859. doi:10.1248/yakushi.129.855

[24] Zhu, H.F., Wan, D., Luo, Y., Zhou, J.L., Chen, L. and Xu, X.Y. (2010) Catalpol increases brain angiogenesis and up-regulates VEGF and EPO in the rat after permanent middle cerebral artery occlusion. International Journal of Biological Sciences, 6, 443-453. doi:10.7150/ijbs.6.443

[25] Nocerino, E., Amato M. and Izzo, A.A. (2000) The aphrodisiac and adaptogenicproperties of ginseng. Fitoterapia, 71, S1-S5. doi:10.1016/S0367-326X(00)00170-2

[26] Tsukioka, Y., Nakao, K., Moriyama, K., Shintani, T., Samukawa, K., Itho, K., Takeshita, F. and Matsuda, H. (2008) Pharmacological study on panax ginseng C. A. Meyer. XVI. Improving effects of Red Ginseng on peripheral circulation disorder. Journal of Traditional Medi- 
cine, 25, 43-51.

[27] Jaenicke, B., Kim, E.J., Ahn, J.W. and Lee, H.S. (1991) Effect of Panax ginseng extract on passive avoidance retention in old rats. Archives of Pharmacal Research, 14, 25-29. doi:10.1007/BF02857809

[28] Kimura, Y. and Yanagimachi, R. (1995) Intracytoplasmic sperm injection in the mouse. Biology of Reproduction, 52, 709-720. doi:10.1095/biolreprod52.4.709

[29] Petersen, C.G., Massaro, F.C., Mauri, A.L., Oliveira, J.B., Baruffi, R.L. and Franco Jr., J.G. (2010) Efficacy of hyaluronic acid binding assay in selecting motile sperm atozoa with normal morphology at high magnification. Reproductive Biology and Endocrinology: RB\&E, 8, 149.

[30] Yokota, Y. and Sakamoto, H. (2005) Essential manual of assisted reproductive technology for embryologists. In:
Suzuki, S., Hukuda, A., Araki, Y. and Sato, K., Eds., Luteal Support. Ishiyaku Publishers, Tokyo, 353-356.

[31] Kuwayama, M. (2007) Highly efficient vitrification for cryopreservation of human oocytes and embryos: The Cryotop method. Theriogenology, 67, 73-80. doi:10.1016/j.theriogenology.2006.09.014

[32] Rienzi, L., Ubaldi, F., Anniballo, R., Cerulo, G. and Greco, E. (1998) Preincubation of humanoocytes may improve fertilization and embryo quality after intracytoplasmic sperm injection. Human Reproduction, 13, 1014 1019. doi:10.1093/humrep/13.4.1014

[33] Iwahata, H., Endoh, S. and Hirai, Y. (2006) Treatment of female infertility incorporating low-reactive level laser therapy (LLLT): An initial report. Laser Therapy, 15, 3741. doi: $10.5978 /$ islsm. 15.37 